\title{
Some numerical simulations of pseudoelastic hysteresis in shape memory alloys
}

\author{
Martin/Brokate \\ Fachbereich Mathematik \\ Universität Kaiserslautern \\ 6750 Kaiserslautern \\ Germany
}

\author{
Jürgen Theel \\ Fachbereich Mathematik \\ Universität Kaiserslautern \\ 6750 Kaiserslautern \\ Germany
}

\begin{abstract}
Recently, Fedelich and Zanzotto have developed a model for the nonisothermal pseudoelastic behaviour of a shape memory material and have conducted some numerical simulation experiments. We present a different method for the numerical solution and discuss it in comparison with their results.
\end{abstract}

\section{Introduction}

The mathematical analysis and numerical simulation of nonisothermal behaviour of shape memory alloys has received considerable attention during the last ten years. Various models have been proposed and investigated; we refer to [6] for a survey and references concerning the model of Falk and the model of Frémond. Here we concentrate upon the model presented in [4] and extended in [1], where the authors try to explain the pseudoelastic stress-strain behaviour of shape memory alloys actually observed in a certain range of temperature and strain. While the isothermal lumped version of [4] has been confirmed experimentally, see [4] and [2], it is difficult to conduct meaningful experiments for the nonisothermal case, especially if one also wants to include spatial effects as in [1]. On the other hand, a mathematical analysis of the model of [1] is confronted with serious problems due to the discontinuous nature of the memory mechanism (not the actual stress-strain curves; they are continuous) which manifests itself as a discontinuity of the corresponding hysteresis operator. Under these circumstances, numerical simulations appear as a particularly attractive tool to gain some insight into the model, and consequently they have been undertaken in [1]. However, if one interprets the numerical results in such a situation, it is not obvious how to distinguish effects due to the model and effects due to the specific numerical method used for the simulation. We therefore decided that it would be interesting to conduct simulations with a different numerical method and to compare the results. 


\section{The model}

We review the material law as it finally emerges from the references [4], [2] and [1], without attempting to retrace its development from thermomechanical principles or to explain the physical meaning of all parameters. We consider a piece of a two phase (austenite and martensite) shape memory material in the isothermal setting of [4]. Assuming a given constant temperature $T$, the material law relates the macroscopic scalar stress $\sigma$, strain $\epsilon$ and phase fraction $z$ of the martensite phase,

$$
0 \leq z \leq 1
$$

as functions of time in the following way. First, all three variables are connected through the linear equation (we preserve the notation of [1] for the constants)

$$
\sigma=K \epsilon-K \Delta z
$$

so the elasticity modulus $K>0$ represents the slope and the constant $\Delta>0$ the horizontal width of the outer hysteresis loop delineating the region $\Omega(T)$ of pseudoelasticity in Figure 1. The horizontal parts of the boundary of $\Omega$ are given by the upper and lower

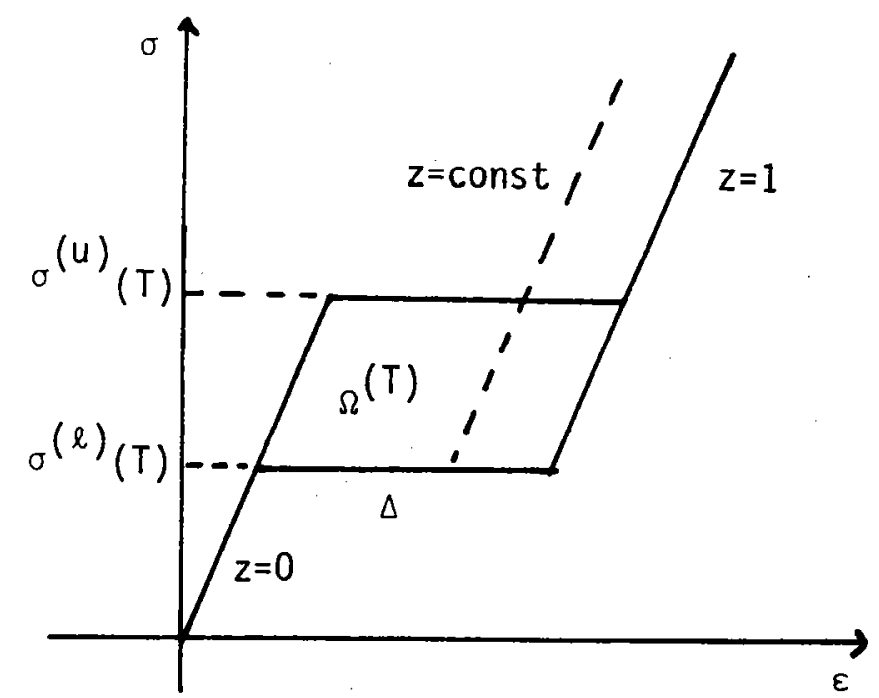

Figure 1: The pseudoelastic region.

values

$$
\sigma^{(u)}(T)=\frac{B(T)+A}{\Delta}, \quad \sigma^{(l)}(T)=\frac{B(T)-A}{\Delta} .
$$

Here, $2 A$ represents the area of the hysteresis loop $\Omega(T)$, and

$$
B(T)=N T+Q
$$

with given constants $N$ and $Q$, fixes its vertical position according to the given temperature $T$. Outside of $\Omega(T)$, only pure states are allowed, namely austenite for $\sigma<\sigma^{(l)}$ and martensite for $\sigma>\sigma^{(u)}$, so

$$
z=0 \text { if } \sigma<\sigma^{(l)}, \quad z=1 \text { if } \sigma>\sigma^{(u)} .
$$


Together, these restrictions define the set $\Sigma(T)$ of admissible states $(\sigma, \epsilon, z)$ in $\mathbf{R}^{3}$ as

$$
\Sigma(T)=\left\{(\sigma, \epsilon, z) \in \mathbf{R}^{3}:(1),(2) \text { and (5) hold }\right\} .
$$

The material law in the interior of $\Omega(T)$ is based upon a memory mechanism involving the line of unstable phase equilibrium $L(T)$ which connects the upper left and the lower right corner of $\Omega(T)$. Actually, any stress-strain evolution is accompanied by an evolution of the actual upper and lower yield stresses $\bar{\sigma}$ and $\underline{\sigma}$ determined with the aid of the line $L(T)$; between these values, the phase fraction is metastable, i.e. $z$ remains constant. A precise formulation follows. As functions of $z$ and $T$, the values of stress and strain along $L(T)$ are given by

$$
\sigma_{e}(z, T)=\sigma^{(u)}(T)-\frac{2 A}{\Delta} z, \quad \epsilon_{e}(z, T)=\frac{K \Delta^{2}-2 A}{K \Delta} z+\frac{\sigma^{(u)}(T)}{K} .
$$

Accordingly, the set $\Sigma(T)$ of admissible states splits into its upper part $\Sigma^{(u)}(T)$ and lower part $\Sigma^{(l)}(T)$,

$$
\begin{aligned}
& \Sigma^{(u)}(T)=\left\{(\sigma, \epsilon, z) \in \Sigma(T): \epsilon>\epsilon_{e}(z, T)\right\} \\
& \Sigma^{(l)}(T)=\left\{(\sigma, \epsilon, z) \in \Sigma(T): \epsilon<\epsilon_{e}(z, T)\right\} .
\end{aligned}
$$

We introduce the piecewise constant memory variable $z_{L}$ with values in $[0,1]$. Every time we cross the line $L(T), z_{L}$ is updated to the value of $z$ at that crossing, and it is kept constant otherwise. We now describe the time evolution. Let an admissible state $P_{*}=\left(\sigma_{*}, \epsilon_{*}, z_{*}\right)$ together with the memory $z_{L_{*}}$ be given. The experimental results of [2] and [4] suggest that a monotone change in either $\sigma$ or $\epsilon$ results in a new admissible state $(\sigma, \epsilon, z)$ on the curve depicted in figure 2. In figure 2 we assume that $P_{*} \in \Sigma^{(u)}(T)$. The

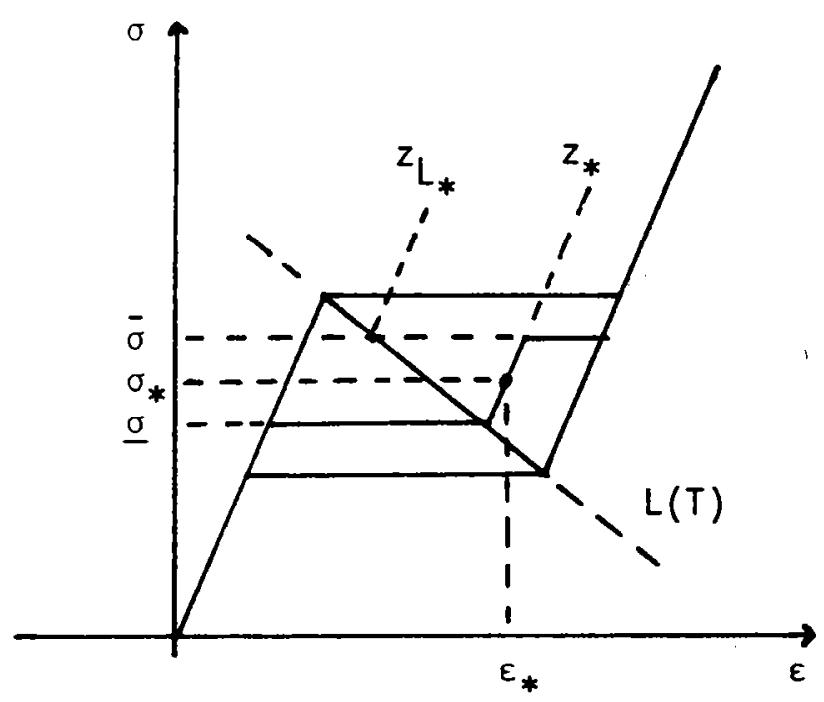

Figure 2: The pseudoelastic material law

case where $P_{*} \in \Sigma^{(l)}(T)$ is analogous. In the former case the current upper and lower yield stress are

$$
\bar{\sigma}=\max \left\{\sigma_{*}, \sigma_{e}\left(z_{L_{*}}, T\right)\right\}, \quad \underline{\sigma}=\sigma_{e}\left(z_{*}, T\right),
$$


and the possible new values of the state $P=(\sigma, \epsilon, z)$ and the memory $z_{L}$ are completely described by the six alternatives

$$
\begin{aligned}
& \sigma>\bar{\sigma}, \quad z=1, \quad z_{L}=z_{L *} \\
& \sigma=\bar{\sigma}, \quad z_{*} \leq z \leq 1, \quad z_{L}=z_{L *} \\
& \sigma_{*} \leq \sigma<\bar{\sigma}, \quad z=z_{*}, \quad z_{L}=z_{L *} \\
& \underline{\sigma}<\sigma \leq \sigma_{*}, \quad z=z_{*}, \quad z_{L}=z_{L *} \\
& \sigma=\underline{\sigma}, \quad 0 \leq z \leq z_{*}, \quad z_{L}=z_{*} \\
& \sigma<\underline{\sigma}, \quad z=0, \quad z_{L}=z_{*} .
\end{aligned}
$$

Corresponding formulas hold in the case $P . \in \Sigma^{(l)}$; to get them, the reader may easily modify (9) - (15) according to figure 2 aind the foregoing discussion.

In both cases, we say that the state $P=(\sigma, \epsilon, z)$ with memory $z_{L}$ is monotonely connected to the state $P_{*}=\left(\sigma_{*}, \epsilon_{*}, z_{*}\right)$ with the memory $z_{L_{*}}$. Performing steps of this type one after the other, we obtain the discrete evolution associated to the pseudoelastic material law.

Definition 2.1 Let $P^{i}=\left(\sigma^{i}, \epsilon^{i}, z^{i}\right), 0 \leq i \leq n$, be admissible states with memory $z_{L}^{i}$, where $P^{0}=(0,0,0)^{1}$. We say that $\left(P^{i}, z_{L}^{i}\right)$ is an admissible discrete evolution, if, for $0<i \leq n$, the state $P^{i}$ with memory $z_{L}^{i}$ is monotonely connected to the state $P^{i-1}$ with memory $z_{L}^{i-1}$.

We can immediately extend this definition to continuous, piecewise monotone processes.

Definition 2.2 Let $\sigma, \epsilon, z:\left[0, t_{f}\right] \rightarrow \mathbf{R}$ be continuous and piecewise monotone with monotonicity partition $\left(t_{i}\right), 0 \leq i \leq n$, let $\sigma(0)=\epsilon(0)=z(0)$. We say that the functions $\sigma, \epsilon, z$ describe an admissible piecewise monotone evolution, if, for any $t \in\left[0, t_{f}\right]$, say $t \in$ $\left[t_{k}, t_{k+1}\right]$, the sequence $(\sigma(0), \epsilon(0), z(0)), \ldots,\left(\sigma\left(t_{k}\right), \epsilon\left(t_{k}\right), z\left(t_{k}\right)\right),(\sigma(t), \epsilon(t), z(t))$ together with some memory $z_{L}^{0}, \ldots, z_{L}^{k}, z_{L}^{t}$ is an admissible discrete evolution.

For deformation controlled experiments with constant temperature $T$, we may rephrase these definitions in operator terminology. It is easy to see that, to any given sequence of strains $0=\epsilon^{0}, \epsilon^{1}, \ldots, \epsilon^{n}$, there corresponds a unique admissible discrete evolution $\left(P^{i}=\left(\sigma^{i}, \epsilon^{i}, z^{i}\right), z_{L}^{i}\right)$. Let now $\epsilon:\left[0, t_{f}\right] \rightarrow \mathbf{R}$ be a continuous, piecewise monotone function with monotonicity partition $\left(t_{i}\right)$. We set $\epsilon^{i}=\epsilon\left(t_{i}\right)$ and define the corresponding discrete evolution. We then can obtain continuous, piecewise monotone functions $\sigma, z$ : $\left[0, t_{f}\right] \rightarrow \mathbf{R}$ in a unique manner such that $\sigma, \epsilon, z:\left[0, t_{f}\right] \rightarrow \mathbf{R}$ is an admissible piecewise monotone evolution. This yields a hysteresis operator $W=\left(W_{\sigma}, W_{z}\right)$,

$$
\sigma(t)=\left(W_{\sigma} \epsilon\right)(t), \quad z=\left(W_{z} \epsilon\right)(t)
$$

such that $W_{\sigma}$ and $W_{z}$ both map a certain subset of $C_{p m}\left[0, t_{f}\right]^{2}$ to $C_{p m}\left[0, t_{f}\right]$. This completes the description of the pseudoelastic constitutive law for an arbitrary, but constant temperature.

Let now the temperature $T$ vary. One has to specify the temperature dependence of the memory mechanism. (This is the main point, since the metastable curves given by (2)

\footnotetext{
${ }^{1}$ This restriction is immaterial and made solely for notational convenience.

${ }^{2} C_{p m}\left[0, t_{f}\right]$ denotes the space of continuous, piecewise monotone functions on $\left[0, t_{f}\right]$.
} 
for constant $z$ do not change with temperature.) As it is shown in [1], one can do this in a natural way for the deformation controlled case, starting from the isothermal situation. To this end, fix a reference temperature $T_{r e s}$. Assume that functions $T, \epsilon \in C_{p m}\left[0, t_{f}\right]$ are given. Consider the auxiliary input function

$$
v(t)=\epsilon(t)-\frac{N}{K \Delta}\left(T(t)-T_{r e f}\right)
$$

set

$$
w(t)=\left(W_{\sigma} v\right)(t), \quad z(t)=\left(W_{z} v\right)(t)
$$

where $W=\left(W_{\sigma}, W_{z}\right)$ is the hysteresis operator given in (16), taken for the reference temperature $T_{r e f}$, and define

$$
\sigma(t)=w(t)+\frac{N}{\Delta}\left(T(t)-T_{r e f}\right) .
$$

In this manner one obtains a nonisothermal hysteresis operator which maps the time evolution of $T$ and $\epsilon$ to the time evolution of $\sigma$ and $z$.

Now consider a quasistatic evolution in one space dimension. The corresponding partial differential equations have been derived in [1] as

$$
\begin{aligned}
\sigma_{x} & =0, \\
F(\epsilon, z) z_{t}+M T_{t}-\kappa T_{x x} & =0,
\end{aligned}
$$

where $M$ and $\kappa$ are constants and

$$
F(\epsilon, z)=A-K \Delta \epsilon+Q+\left(K \Delta^{2}-2 A\right) z
$$

In addition, at any space point $x$ the pseudoelastic material law described above relates the functions $T(x, \cdot), \epsilon(x, \cdot), \sigma(x, \cdot)$ and $z(x, \cdot)$. We remark that for the simulation experiments described later we always have

$$
F(\epsilon, z)<0,
$$

so an increase of the phase fraction $z$, i.e. a transition from austenite to martensite, produces heat, whereas the opposite transition consumes heat.

A mathematical analysis of the system (20) coupled with the hysteresis operator $W$ of (16) is nonexistent. It is complicated by the fact that $W$ is a discontinuous operator. We do not contribute to this problem here.

\section{The basic algorithm}

We want to solve numerically the system (20) coupled to the pseudoelastic material law and complemented by initial and boundary conditions. The material law creates problems, since it introduces discontinuities into the model due to its memory structure and due to the piecewise smoothness of the curves in figure 2. Moreover, the effect of these discontinuities may be different for stress induced and for temperature induced phase transitions. We therefore decided to use as simple and explicit a method as possible, adapted to these two situations, in order to be able to interpret the results and compare them with [1]. 
We describe the underlying discretization and structure of the algorithm common to both situations. We consider a one dimensional bar of length $L$ with $J$ interior discrete space points $x_{j}$,

$$
x_{j}=\left(j-\frac{1}{2}\right) \delta x, \quad \delta x=\frac{L}{J}, \quad 0 \leq j \leq J+1
$$

and fix a discrete time step $\delta t>0$. The discrete values of the various variables at the points $\left(x_{j}, t_{n}\right)$, where $t_{n}=n \Delta t$, are denoted by $\epsilon_{j}^{n}, z_{j}^{n}, T_{j}^{n}, z_{L_{j}}^{n}$ and $\sigma^{n}$. Note that the stress does not depend on $j$. To avoid numerical instability, one of course has to discretize the equation for the temperature with an implicit scheme. We use the standard formula

$$
F\left(z_{j}^{n}, \epsilon_{j}^{n}\right) \frac{z_{j}^{n+1} \ldots \tilde{y}_{j}^{n}}{\delta t}+M \frac{T_{j}^{n+1}-T_{j}^{n}}{\delta t}-\kappa \frac{T_{j+1}^{n+1}-2 T_{j}^{n+1}+T_{j-1}^{n+1}}{(\delta x)^{2}}=0 .
$$

Basic algorithm. Assume that $\epsilon_{j}^{n}, z_{j}^{n}, T_{j}^{n}, z_{L, j}^{n}, \sigma^{n}$ are given. (For $n=0$ use the initial conditions.) Then perform the next time step as follows:

- Stress-strain step: Compute $\epsilon_{j}^{n+1}, z_{j}^{n+1}, z_{L, j}^{n+1}, \sigma^{n+1}$ from the pseudoelastic material law with fixed temperature $T_{j}^{n}$.

- Temperature step: Compute $T_{j}^{n+1}$ as a solution of (24), complemented by the boundary conditions.

In this manner, we decompose the time step into an only "slightly" implicit step (to be described in the next sections) involving the nonlinear material law and the memory update, and an implicit linear step. The latter reduces to the solution of a linear tridiagonal system which requires only $O(J)$ operations.

\section{Simulation of a stress induced phase transition}

We start with an unloaded specimen at constant temperature $T_{0}$, so the initial conditions are

$$
\epsilon(x, 0)=\sigma(x, 0)=z(x, 0)=0, \quad T(x, 0)=T_{0} .
$$

We keep the boundary temperature constant,

$$
T(0, t)=T(L, t)=T_{0},
$$

fix the left end of the bar at 0 and control the displacement $U(t)$ of the right end, so $\epsilon$ has to satisfy

$$
U(t)=\int_{0}^{L} \epsilon(x, t) d t .
$$

For the computation, the control function $U$ is replaced by discrete values $U^{n}=U\left(t_{n}\right)$. We now describe how the stress-strain step from $t_{n}$ to $t_{n+1}$ in the basic algorithm of the preceding section is carried out. The discrete equations to be satisfied at $t=t_{n+1}$ are

$$
U^{n+1}=\delta x \sum_{j=1}^{J} \epsilon_{j}^{n+1},
$$




$$
\begin{gathered}
\epsilon_{j}^{n+1}=\frac{1}{K^{-}} \sigma^{n+1}+\Delta z_{j}^{n+1}, \quad 1 \leq j \leq J, \\
\sigma^{n+1}=W_{\sigma, j}^{n+1}\left(\epsilon_{j}^{n+1} ; \sigma^{n}, z_{j}^{n}, T_{j}^{n}, z_{L, j}^{n}, v_{j}^{n}\right), \quad 1 \leq j \leq J .
\end{gathered}
$$

These are $2 J+1$ equations with the same number of unknowns. The map $W_{\sigma, j}^{n+1}$ has to realize the monotone connection from the state $\left(\sigma^{n}, \epsilon_{j}^{n}, z_{j}^{n}\right)$ to the state $\left(\sigma^{n+1}, \epsilon_{j}^{n+1}, z_{j}^{n+1}\right)$ in the current set $\Sigma\left(T_{j}^{n}\right)$ of admissible states, given the memory $z_{L, j}^{n}$. We have also added for convenience the memory variable $v_{j}^{n}$, which has the value 1 if the previous stressstrain step ended in the region $\Sigma^{(u)}\left(T_{j}^{n-1}\right)$; i.e. above the unstable phase equilibrium, and -1 if it ended in $\Sigma^{(l)}\left(T_{j}^{n-1}\right)$.

In the numerical algorithm, one has to specify (explicitly or implicitly) how the temperature variation interacts with the memory. We decided that the memory values $z_{L, j}^{n}$ should be updated only as a result of the stress-strain step, but not after the temperature step. This is natural, since we want to model stress-induced phase transitions, and actually helps to avoid certain numerical oscillations. However, due to the movement of the region $\Omega(T)$ in the temperature step, the state $\left(\sigma^{n}, \epsilon_{j}^{n}, z_{j}^{n}\right)$ with memory $z_{L, j}^{n}$ may not be compatible with the position of $\Omega\left(T_{j}^{n}\right)$. We therefore modify slightly the definition of the current upper and lower yield stress in (9). In the case $v_{j}^{n}=1$ (the other is analogous), we set

$$
\bar{\sigma}_{j}^{n}=\max \left\{\sigma^{n}, \sigma_{e}\left(z_{L, j}^{n}, T_{j}^{n}\right)\right\}
$$

even if then $\bar{\sigma}_{j}^{n}$ exceeds $\sigma^{(u)}\left(T_{j}^{n}\right)$ and we are outside $\Omega\left(T_{j}^{n}\right)$, and

$$
\underline{\sigma}_{j}^{n}=\min \left\{\sigma^{n}, \sigma_{e}\left(z_{j}^{n}, T_{j}^{n}\right)\right\}
$$

(After reading the following paragraphs, the reader easily checks that with these modifications, the algorithm will restore admissibility as quickly as possible.) For a given value $\epsilon_{j}^{n+1}$, we can now define the new state $\left(\sigma^{n+1}, \epsilon_{j}^{n+1}, z_{j}^{n+1}\right)$ with the memory $z_{L, j}^{n+1}$ as the state $P$ with memory $z_{L}$ constructed from $P_{*}=\left(\sigma^{n}, \epsilon_{j}^{n}, z_{j}^{n}\right)$ with memory $z_{L *}$ according to $(10)-(15)$, with the upper and lower yield stress given by (31) and (32). We may then interpret (30) as the formal definition of the monotone step map $W_{\sigma, j}^{n+1}$. We next explain how to solve the system (28) - (30). Although it is in principle nonlinear, it can be solved rather efficiently, since the monotone step map $W_{\sigma, j}^{n+1}$ is monotone and piecewise linear with respect to $\epsilon_{j}^{n+1}$. We describe the procedure for the case $U^{n+1}>U^{n}$. In this case we must obviously have $\sigma^{n+1} \geq \sigma^{n}$ and therefore

$$
z_{j}^{n+1} \geq z_{j}^{n}, \quad \epsilon_{j}^{n+1} \geq \epsilon_{j}^{n}
$$

for all $j, 1 \leq j \leq J$. We define $\bar{\sigma}_{j}^{n}$ as in (31) and sort these values in increasing order. We now are at a point where another decision has to be made which has actually been left open in the model of [1]. If the upper yield stresses at two or more discrete space points are equal, it may happen that the solution to (28) is not unique, since there may be various combinations of intermediate phase fractions (and, hence, strains) on the upper yield stress line which give the same sum in (28). (The same ambiguity is present in the continuous formulation.) We decided to remove this ambiguity by requiring that the phase fractions at all such space points have to be equal at the end of the current stress-strain step. We even go one step further and group the space points with almost equal values of $\bar{\sigma}_{j}^{n}$, i.e. we set $\bar{\sigma}_{j}^{n}$ to a common equal value. Without this correction, 
roundoff noise tends to form a large number of phase boundaries in a portion of the bar which enters the pseudoelastic region at approximately the same time and temperature. We return to the main line of argument. We observe that we may rewrite (28) with the aid of (29) as

$$
U^{n+1}=\delta x\left(\frac{J}{K} \sigma^{n+1}+\Delta\left(m^{n+1}+z^{n+1}\right)\right),
$$

where $m^{n+1}$ denotes the number of discrete space points in a pure martensite phase, and $z^{n+1}$ denotes the sum of the phase fractions of all discrete space points in a mixed phase. We now step through the sorted values $\bar{\sigma}_{j}^{n}$ in increasing order until the right side of (34) becomes bigger than the given value $U^{n+1}$. A simple linear interpolation then produces equality in (34). Since at every value we examine, we can easily produce the correct final values of $z_{j}^{n+1}$ and of the memory $z_{L, j}^{n+1}, v_{j}^{n+1}$, the system (28) $-(30)$ can be solved with 5 - 10 flops per space point. This completes the description of the stress-strain step for the boundary displacement controlled simulation.

For the actual computations, we have used the data of [1] estimated for a CuZnAl-alloy, namely

$$
\begin{gathered}
K=10^{4} M P a, \quad N=0.105 \frac{M P a}{K}, \kappa=376 \frac{W}{m K}, \quad Q=-27.48 M P a \\
\Delta=0.06, \quad A=0.3 M P a, \quad L=0.015 \mathrm{~m} \\
M=\rho C, \quad \rho=8.9 \cdot 10^{3} \frac{\mathrm{kg}}{\mathrm{m}^{3}}, \quad C=385 \frac{\mathrm{J}}{\mathrm{kgK}} .
\end{gathered}
$$

We take $J=150$ interior space points and a time step $\delta t=10^{-3}$ seconds. We first consider pure loading

$$
U(t)=0.001 t
$$

and get two phase fronts starting at the lateral boundaries and symmetrically moving inwards, see figure 3. The corresponding temperature profile is shown in figure 4. This is in good agreement with [1], except that we get very sharp phase boundaries. This difference is clearly due to the different numerical methods, since our stress-strain

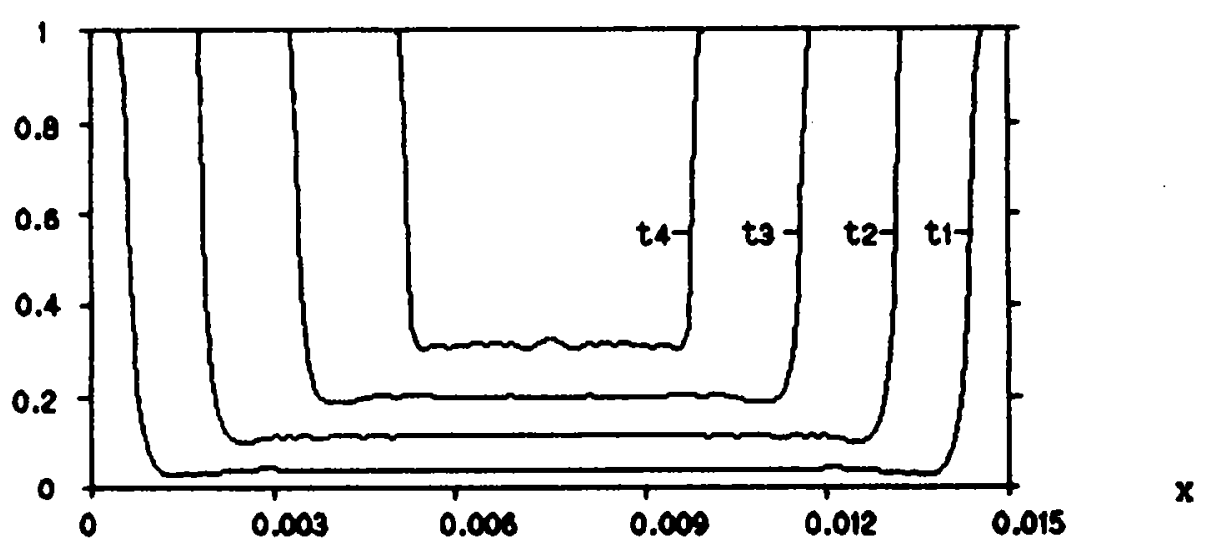

Figure 3: Pure loading; phase fraction as function of $x$.

step produces sharp fronts by clesign, whereas the algorithm presented in [1] tends to introduce smoothing, presumably because of its nested implicit structure. 


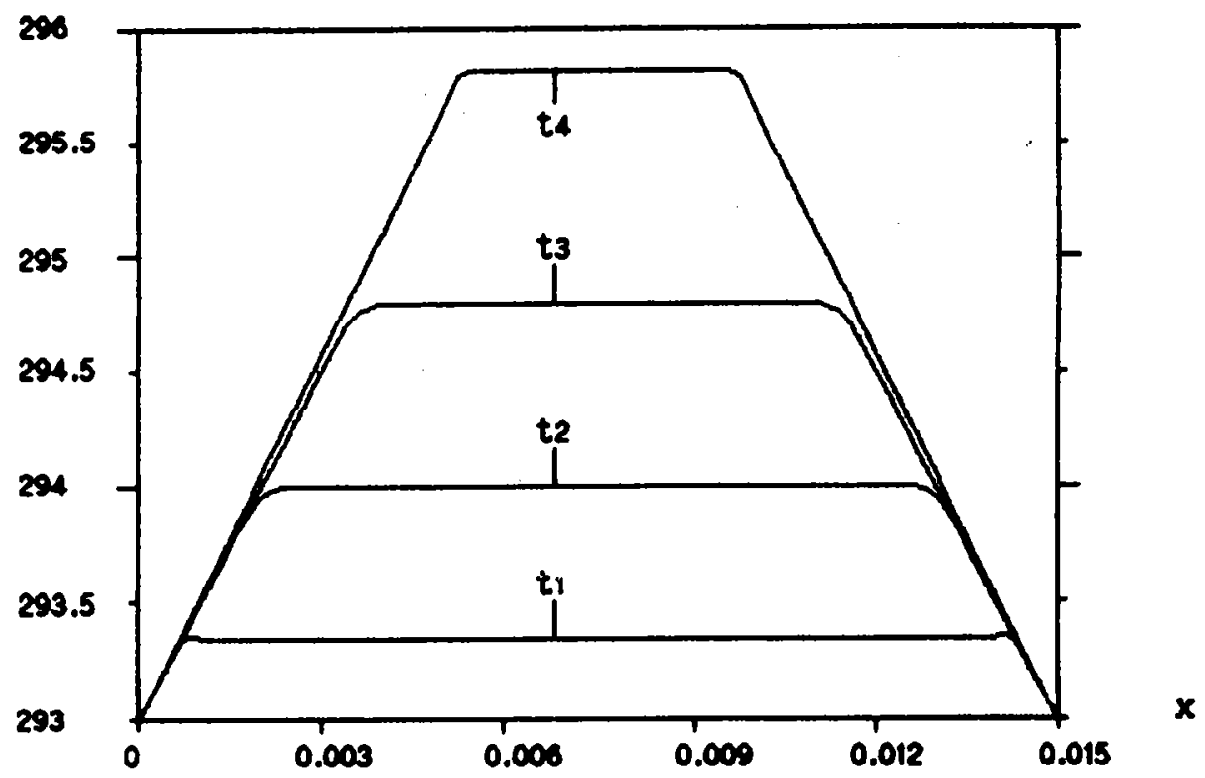

Figure 4: Pure loading, temperature as function of $x$.

For the second simulation, we start as before with the loading (36) until some time $t_{1}$. Then we switch to unloading at the same rate. The results for several later time instances are presented in figures 5 and 6 . During unloading, at first the space points in mixed phase transform back to austenite the moment they hit the unstable phase equilibrium. This occurs in a somewhat irregular sequence due to small fluctuations in temperature probably introduced by the numerical scheme. At time $t_{3}$ this process is completed, and new phase fronts start to move inward from the lateral boundary. We do not observe the martensite needle reported in [1], so that may be an artifact. Except for this and our irregular fluctuations, there is again good qualitative and quantitative agreement with the results of [1].

The third simulation starts off at the point $t_{2}$ of the second simulation, where the space points in mixed phase have developed some irregular structure. We again load, with the same rate as before, in order to force part of the mixed phase points to run through a hysteresis loop within the pseudoelastic region. As is to be expected from the construction of our method, these irregularities are amplified since now the mixed phase points transform to pure martensite again not in a uniform manner, but according to some nonmonotone temperature variation in the middle part of the sample, see the graphs in figures 7 and 8 . This definitively contrasts with the results of [1]. Actually, it is currently under discussion how the number of phase fronts between austenite and martensite is related to the macroscopic pseudoelastic hysteresis loop, and the "irregular" behaviour of our method seems to have some counterpart in experimental observations. Of course, the point here is not to claim that our method is better, but to show how large qualitative differences may arise, if one applies natural, but differently constructed, methods to this problem due to its inherent discontinuities. 

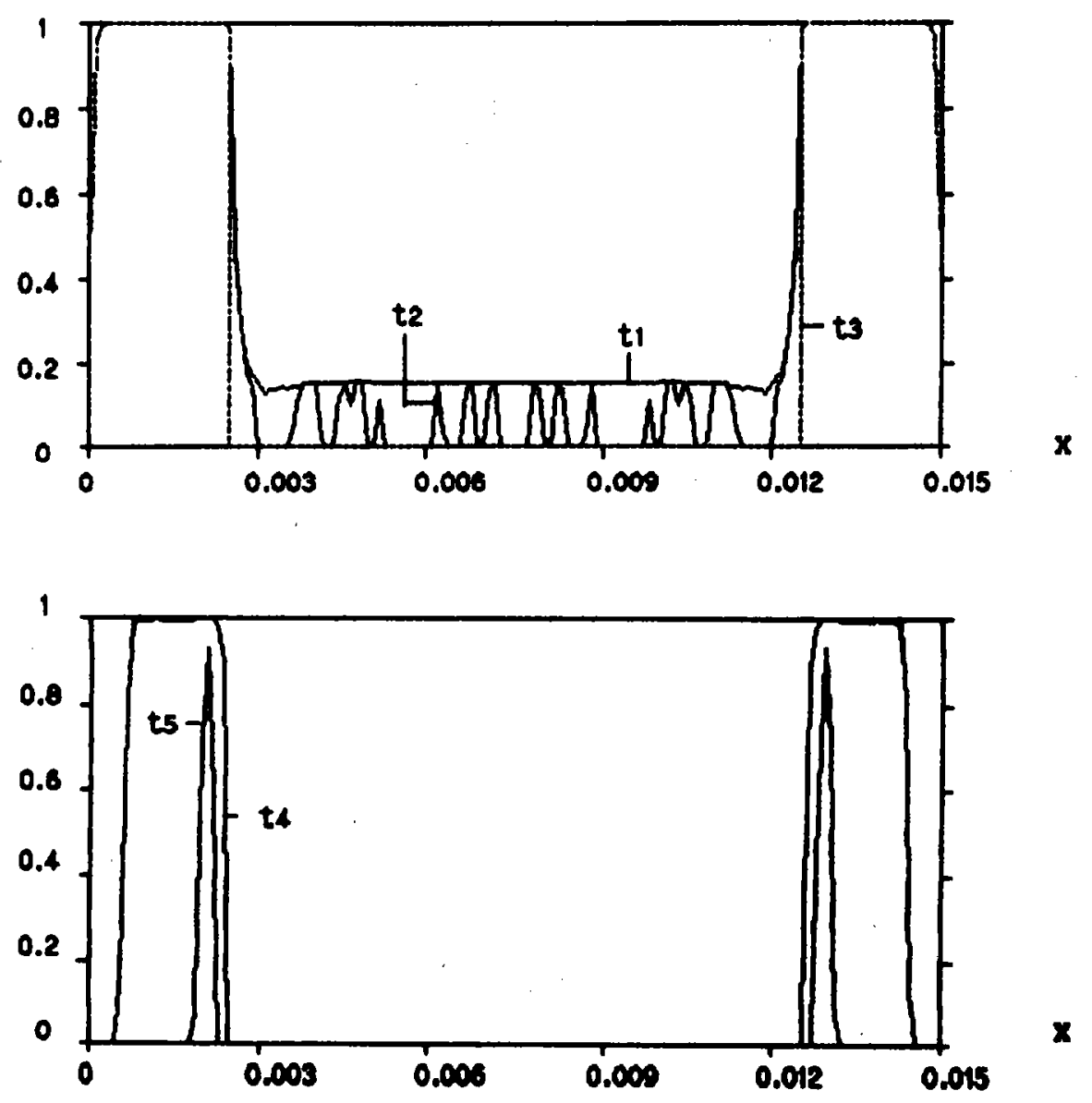

Figure 5: Loading-Unloading, phase fraction as function of $x$.

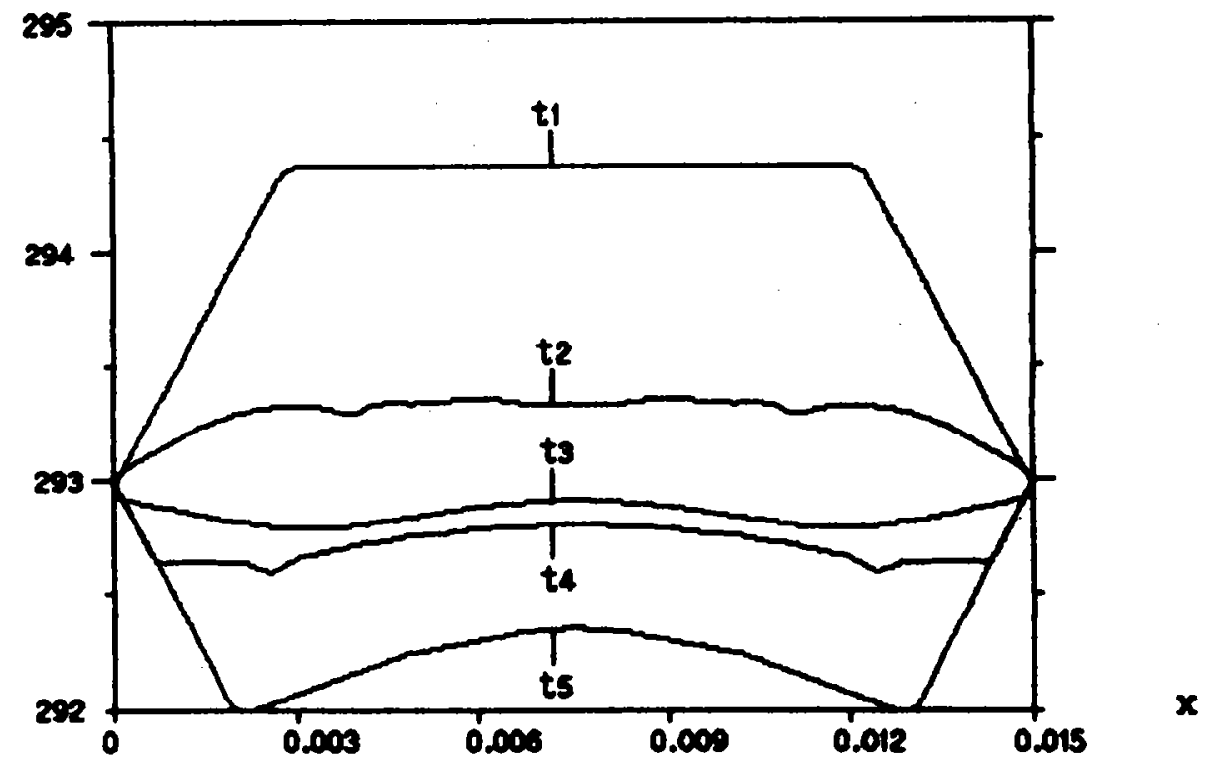

Figure 6: Loading-Unloading, temperature as function of $x$. 

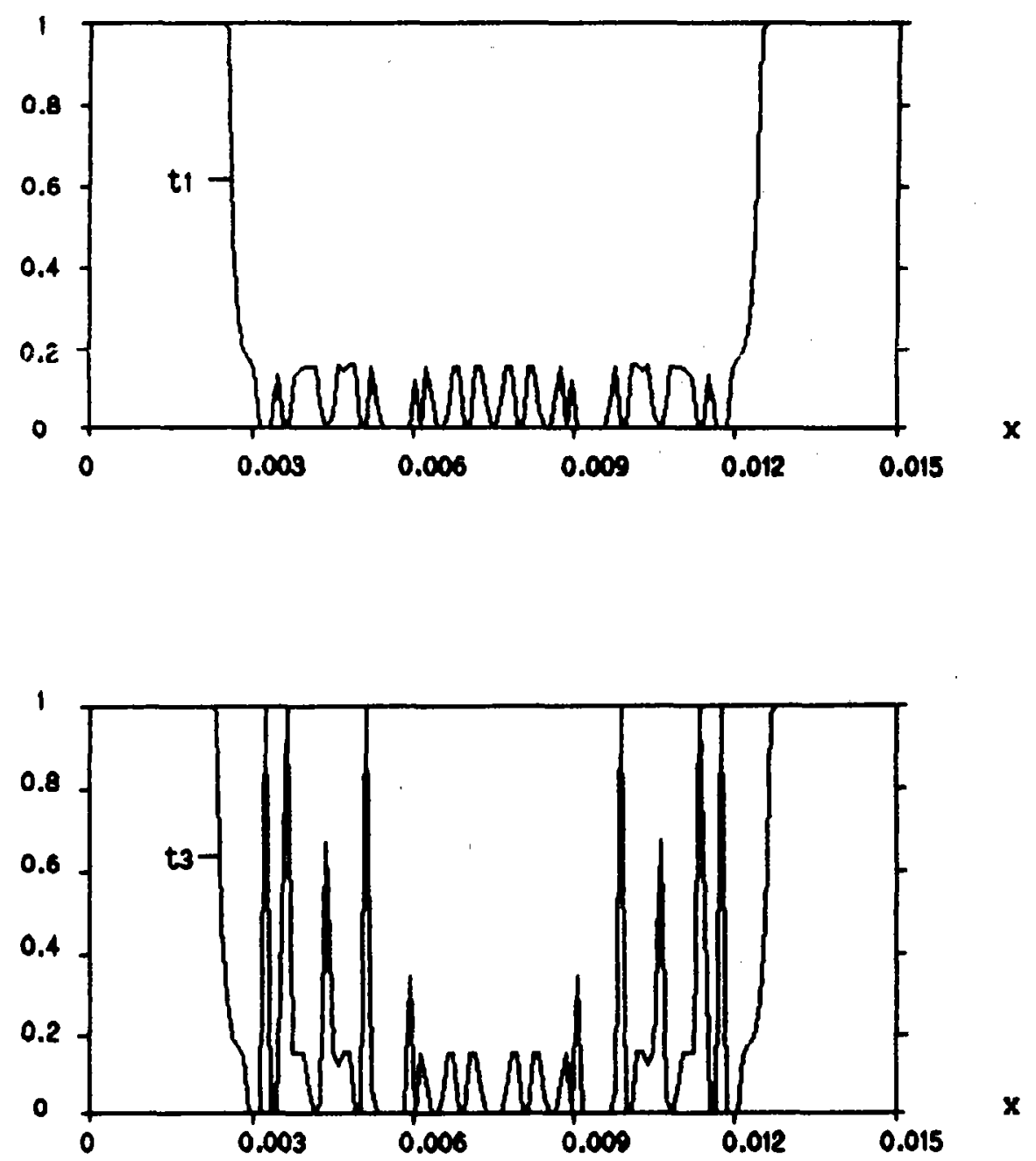

Figure 7: Loading-Unloading-Loading, phase fraction. 

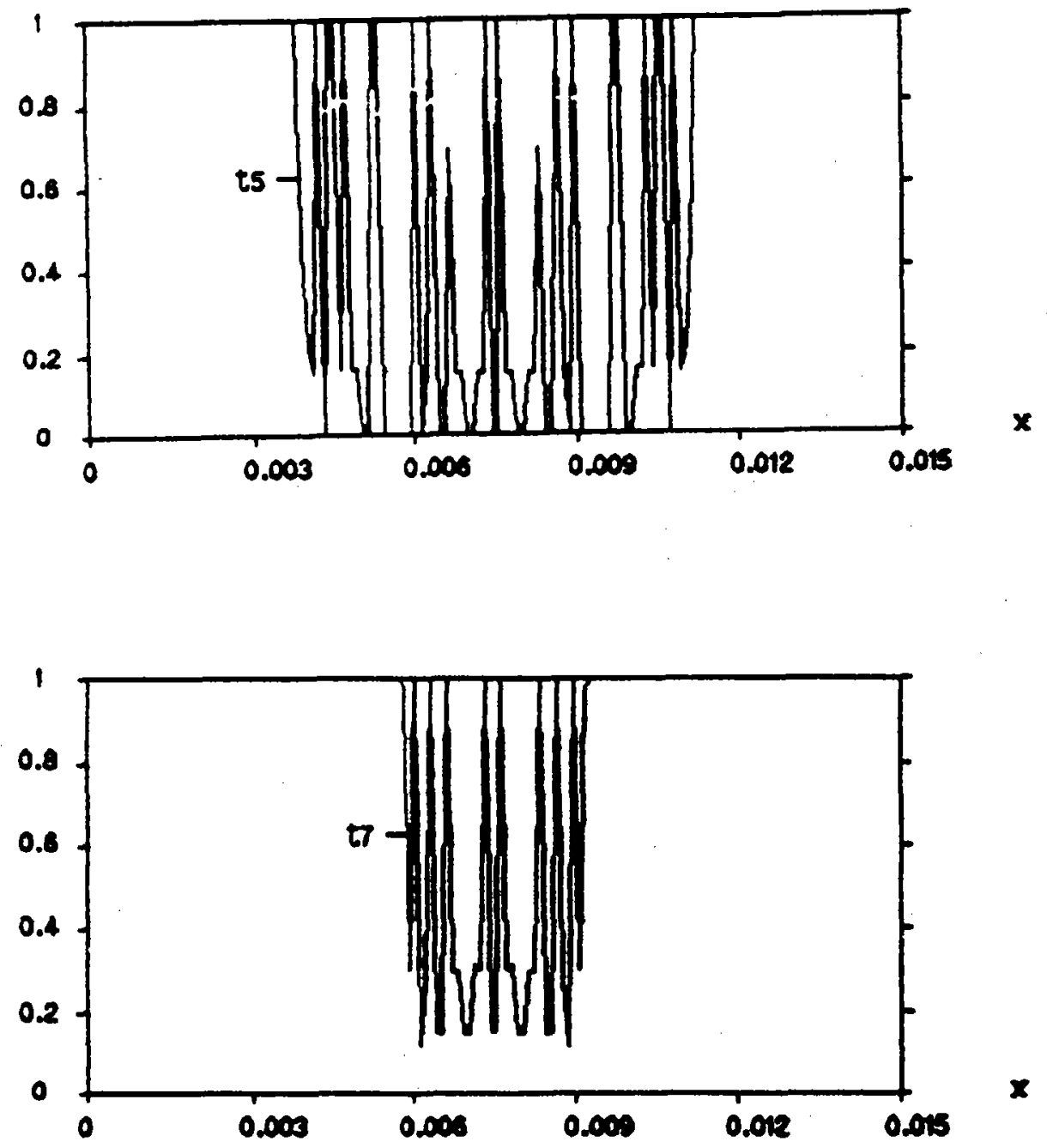

Figure 8: Same as figure $\pi$, later time instances. 


\section{Simulation of a temperature induced phase tran- sition}

We fix the bar at the left end and impose a constant stress $\sigma_{L}$ at the right end. For the quasistatic situation we then have a constant stress

$$
\sigma(x, t)=\sigma_{L}
$$

throughout the whole specinen. As an initial condition we choose

$$
T(x, 0)=T_{0}, \quad z(x, 0)=1, \quad \epsilon(x, 0)=\frac{1}{K} \sigma_{L}+\Delta,
$$

which is compatible with (3T). We control the boundary temperature $\vartheta(t)$,

$$
T(0, t)=\nu(t)=T(L, t) .
$$

We interpret this setting in terms of the material law. Let us fix some space point $x$. At this point, the pseudoelastic region $\Omega(T)$ moves up and down due to the variation of the temperature, while the state stays on the horizontal line $\sigma=\sigma_{L}$. A change in phase fraction and strain occurs only if the current (temperature dependent) yield stress equals $\sigma_{L}$. Again, there is a question of modeling. If the temperature is a strictly monotone function of tine, then so is the current yield stress, and the state immediately jumps to the pure phase when the value $\sigma_{L}$ is crossed. On the other hand, if we imagine that the temperature remains constant for a finite time interval, then it is possible to enter the interior of $\Omega(T)$ and to have a phase mixture at the same point. We did not want to decide this question a priori and implemented the stress-strain step in the basic algorithm in the following way:

Let $\sigma^{n}, \epsilon_{j}^{n}, z_{j}^{n}, T_{j}^{n}, z_{L, j}^{n}$ be given. Compute the current yield stresses $\bar{\sigma}_{j}^{n}$ and $\underline{\sigma}_{j}^{n}$ according to $(9)$. If

$$
\underline{\sigma}_{j}^{n} \leq \sigma_{L} \leq \bar{\sigma}_{j}^{n}
$$

then we are in the metastable situation, and the phase fraction is not changed in this time step. Let us consider the case where $\sigma_{L}<\underline{\sigma}_{j}^{n}$ (the other is treated similarly). We define the new phase fraction by the formula

$$
z_{j}^{n+1}=\frac{1}{\Delta}\left(\epsilon_{j}^{n}-\frac{\sigma_{j}^{n}}{K}\right)
$$

We obtain (41) if in equation (2) at $t=t_{n}$, namely

$$
\sigma_{L}=K \epsilon_{j}^{n}-\Pi \Delta z_{j}^{n},
$$

we replace $\sigma_{L}$ by $\underline{\sigma}_{j}^{n}$. Then we restore (2) at $t=t_{n+1}$ by setting

$$
\epsilon_{j}^{n+1}=\frac{1}{K} \sigma_{L}+\Delta z_{j}^{n+1} \text {. }
$$

In effect, the phase fraction is decreased and the state moves to the left on the line $\sigma=\sigma_{L}$. Since the amount of decrease in a single time step is proportional to the 
difference of $\sigma_{L}$ and $\underline{\sigma}_{j}^{n}$, one can artificially control the time duration of the phase transition at the point $x$ through the time discretization parameter $\delta t$.

For the numerical computation, wo use the same values for the parameters as in the previous section, namcly those given in (35). In the first simulation, we increase the boundary temperature at a rate of $1 \mathrm{~K} / \mathrm{sec}$. This initiates a phase transition from martensite to austenite at the end of the bar, and from each end a phase front moves inward. The space profiles of phase fraction and temperature at subsequent times are shown in figures 9 and 10 for $\delta t=10^{-3}$ and in figures 11 and 12 for $\delta t=10^{-6}$ seconds. As one notices particularly in figure 10 , the heat supply is consumed in the phase transition

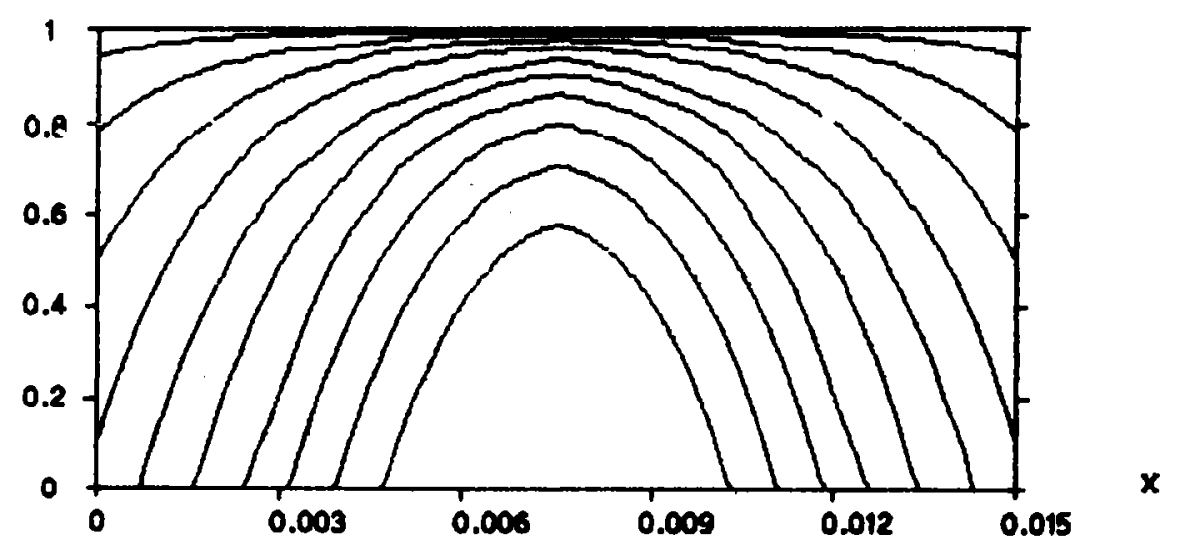

Figure 9: Heating with $\delta t=10^{-3}$, phase fraction as function of $\boldsymbol{x}$.

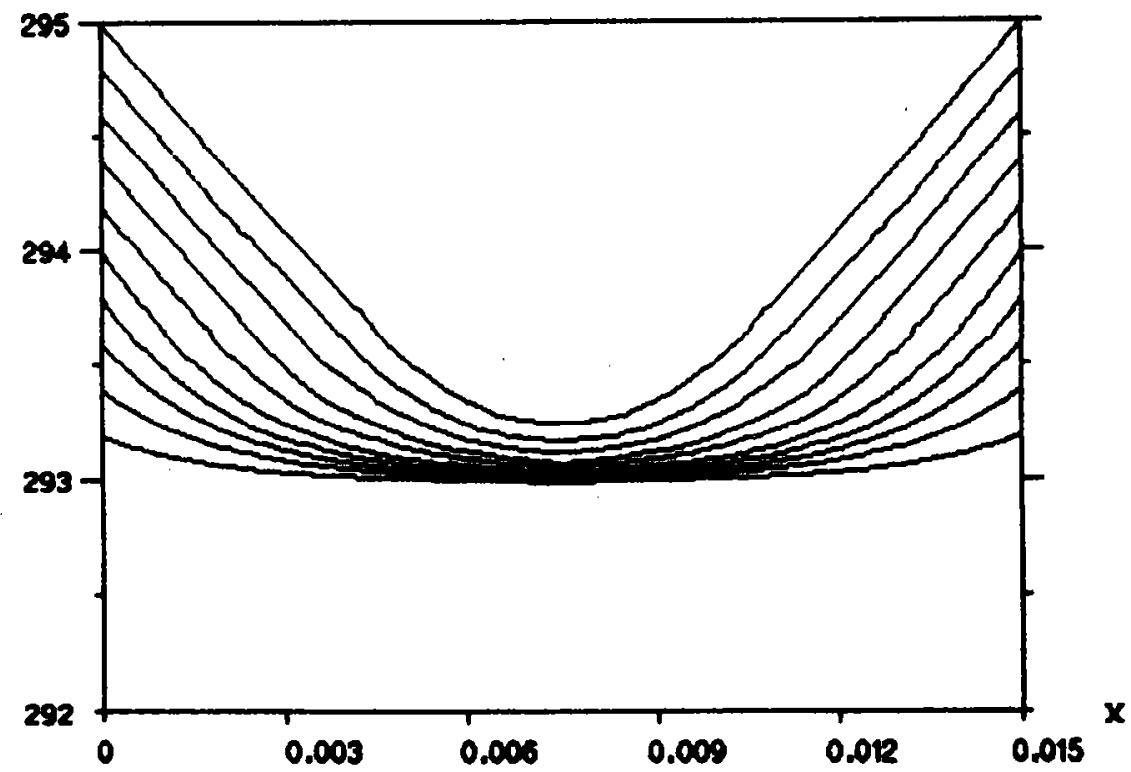

Figure 10: Heating with $\delta t=10^{-3}$, temperature as function of $x$.

(and in warming up the zone between the boundaries and the phase fronts), but no heat reaches the martensite before the pliase transition starts. This agrees with the results 


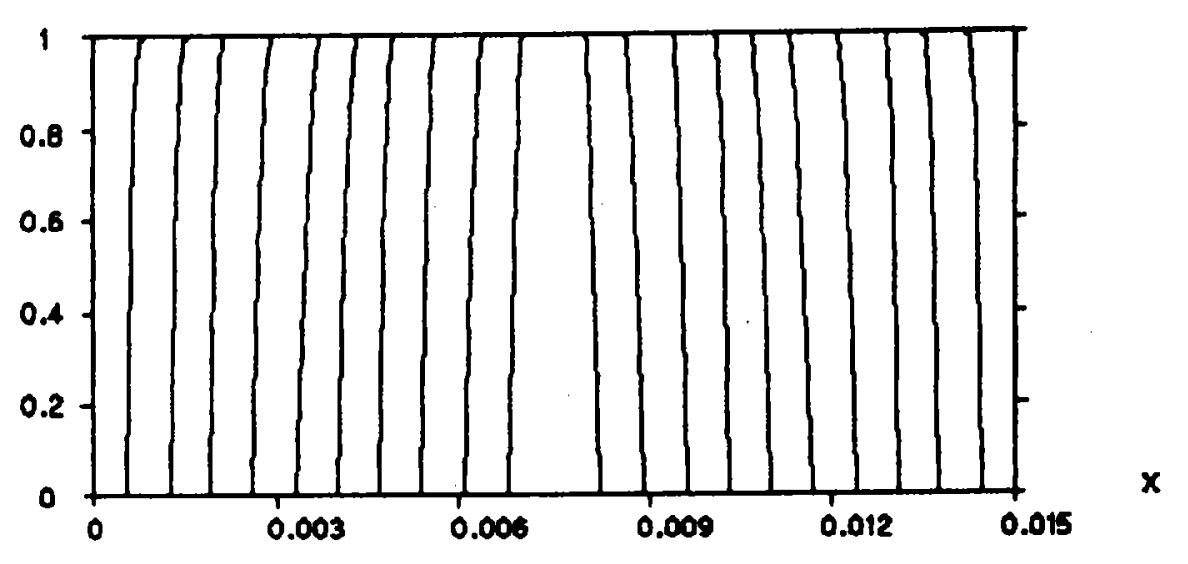

Figure 11: Heating with $\delta t=10^{-6}$, phase fraction as function of $x$.

of [1]. It also turns out that the speed of the phase front does not depend on the time discretization (i.e., it seems to converge as $\delta t$ becomes small), whereas the slopes in the $z$ profile are almost entirely discretization dependent (the front gets sharper as $\delta t$ gets smaller). For different heating rates we obtain the same results. These observations are somewhat in contrast to the interpretation of [1].

We want to present one more simulation. Using the time step $\delta t=10^{-4}$, we heat as before with the constant rate $1 \mathrm{~K} / \mathrm{sec}$ until some time $t_{1}$, and then start to cool at the same rate. The results are shown in figure 13. For some time (until $t=t_{3}$ ) the phase front continues to move inward. Then the cooling makes itself felt and the front starts to move backward. Note that the space points in mixed phase completely transform back to martensite (until $t=t_{6}$ ), before the pure austenites begin to transform. Since we cool at the ends, a second phase front starts to move inwards, while the first front remains more or less fixed. A similar pattern (elimination of mixed phased, then creation of a new front) develops if once again we revert to heating. 


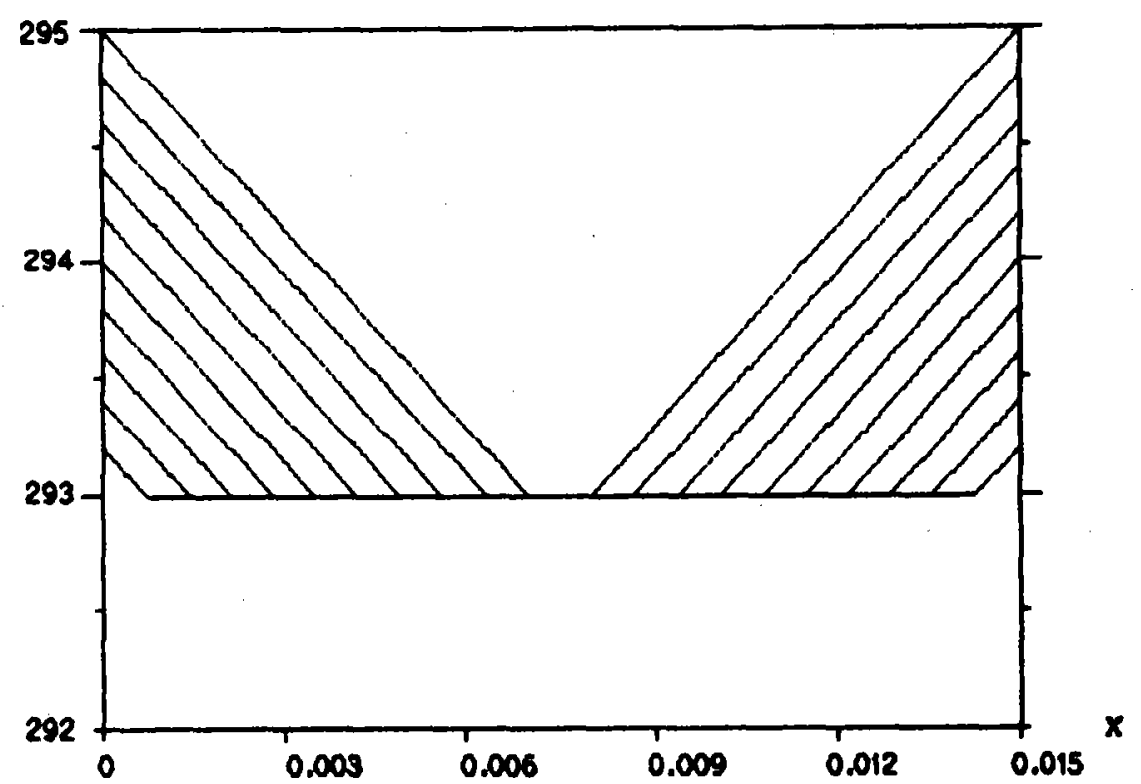

Figure 12: Heating with $\delta t=10^{-6}$, temperature as function of $x$. 

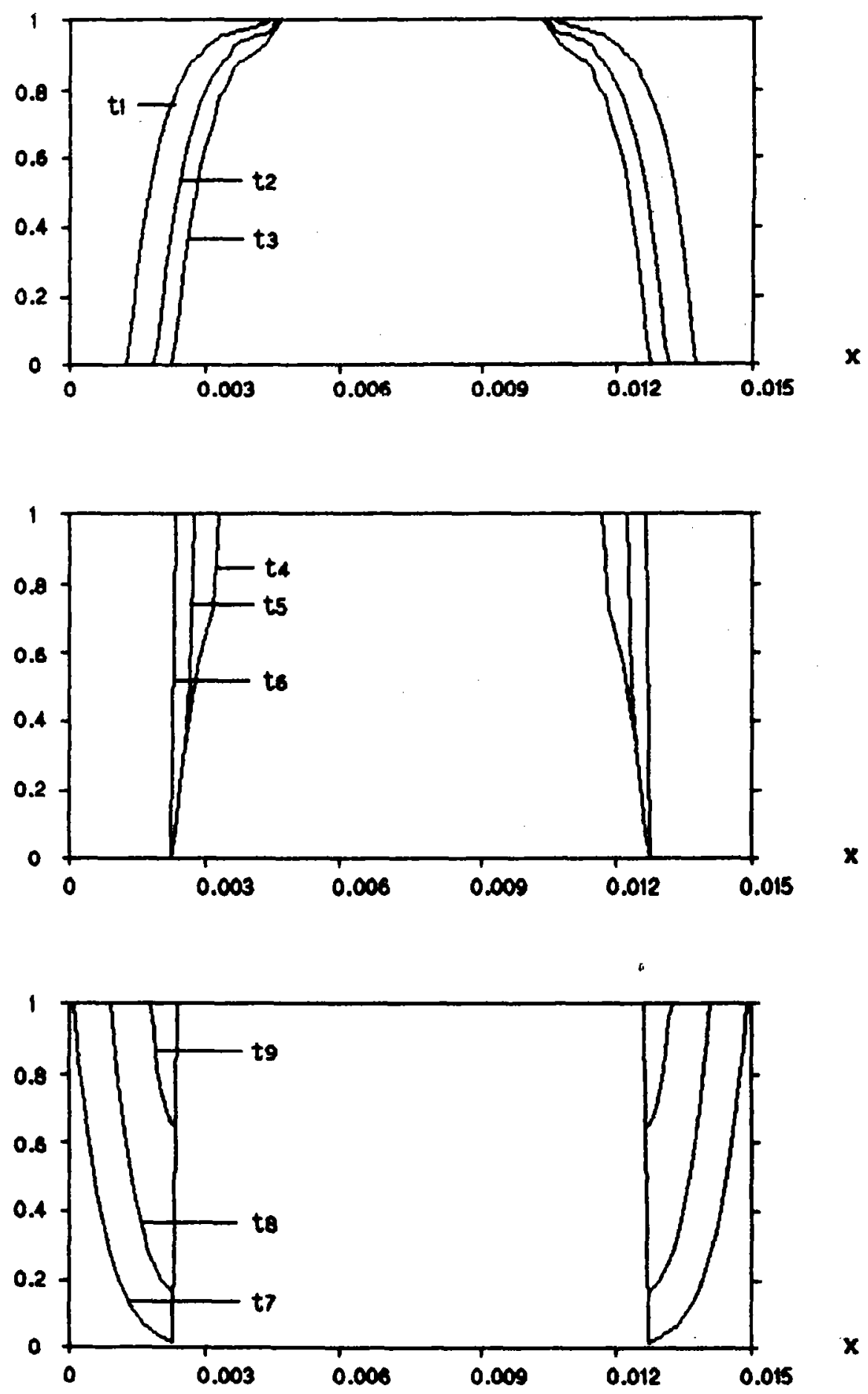

Figure 13: Heating-Cooling, phase fraction as function of $x$. 


\section{References}

[1] Fedelich, B., Zanzotto, G.: One-dimensional quasistatic nonisothermal evolution of shape-memory material inside the hysteresis loop, Continuum Mech. Thermodyn. 3 (1991), $215-270$.

[2] Fu, S., Müller, I., Xu, H.: Experimental and theoretical investigation of the pseudoelastic hysteresis, to appear.

[3] Krasnoselskii, M.A., Pokrovskii, A.V.: Systems with hysteresis, Springer 1989. Russian edition: Nauka, Moscow, 1983.

[4] Müller, I., Xu, H.: On the pseudo-elastic hysteresis, Acta Metall. 39 (1991), 263 271.

[5] Niezgódka, M., Sprekels, J.: Convergent numerical approximations of the thermomechanical phese trunsitions in shape memory alloys, Numer. Math. 58 (1991), 759 $-778$.

[6] Sprekels, J.: Shape memory alloys: Mathematical models for a class of first order solid-solid phase transition in metals, Control and Cybernetics 19(1990), 287 308.

[7] Wilmanski, K.: A model of stress-induced patterns in shape memory alloys, submitted. 